\title{
The Effect of the Molecular Weight of Polyvinylpyrrolidone and the Model Drug on Laser-Induced In Situ Amorphization
}

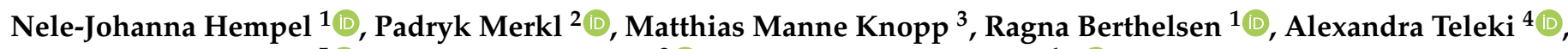 \\ Anders Kragh Hansen ${ }^{5}{ }^{-}$, Georgios A. Sotiriou ${ }^{2}{ }^{-}$and Korbinian Löbmann ${ }^{1, *}$ \\ 1 Department of Pharmacy, University of Copenhagen, 2100 Copenhagen, Denmark; \\ nele.hempel@sund.ku.dk (N.-J.H.); ragna.berthelsen@sund.ku.dk (R.B.) \\ 2 Department of Microbiology, Tumor and Cell Biology, Karolinska Institute, 17177 Stockholm, Sweden; \\ padryk.merkl@ki.se (P.M.); georgios.sotiriou@ki.se (G.A.S.) \\ 3 Bioneer:FARMA, Department of Pharmacy, University of Copenhagen, 2100 Copenhagen, Denmark; \\ mmk@bioneer.dk \\ 4 Science for Life Laboratory, Department of Pharmacy, Uppsala University, 75123 Uppsala, Sweden; \\ alexandra.teleki@scilifelab.uu.se \\ 5 Department of Photonics Engineering, Technical University of Denmark, 4000 Roskilde, Denmark; \\ ankrh@fotonik.dtu.dk \\ * Correspondence: korbinian.loebmann@sund.ku.dk
}

\section{check for}

updates

Citation: Hempel, N.-J.; Merkl, P.; Knopp, M.M.; Berthelsen, R.; Teleki,

A.; Hansen, A.K.; Sotiriou, G.A.;

Löbmann, K. The Effect of the

Molecular Weight of

Polyvinylpyrrolidone and the Model Drug on Laser-Induced In Situ

Amorphization. Molecules 2021, 26,

4035. https://doi.org/

10.3390/molecules26134035

Academic Editor: Josef Jampilek

Received: 10 June 2021

Accepted: 25 June 2021

Published: 1 July 2021

Publisher's Note: MDPI stays neutral with regard to jurisdictional claims in published maps and institutional affiliations.

Copyright: (c) 2021 by the authors. Licensee MDPI, Basel, Switzerland. This article is an open access article distributed under the terms and conditions of the Creative Commons Attribution (CC BY) license (https:// creativecommons.org/licenses/by/ $4.0 /)$.

\begin{abstract}
Laser radiation has been shown to be a promising approach for in situ amorphization, i.e., drug amorphization inside the final dosage form. Upon exposure to laser radiation, elevated temperatures in the compacts are obtained. At temperatures above the glass transition temperature $\left(T_{\mathrm{g}}\right)$ of the polymer, the drug dissolves into the mobile polymer. Hence, the dissolution kinetics are dependent on the viscosity of the polymer, indirectly determined by the molecular weight $\left(M_{\mathrm{W}}\right)$ of the polymer, the solubility of the drug in the polymer, the particle size of the drug and the molecular size of the drug. Using compacts containing $30 \mathrm{wt} \%$ of the drug celecoxib (CCX), $69.25 \mathrm{wt} \%$ of three different $M_{\mathrm{W}}$ of polyvinylpyrrolidone (PVP: PVP12, PVP17 or PVP25), $0.25 \mathrm{wt} \%$ plasmonic nanoaggregates (PNs) and $0.5 \mathrm{wt} \%$ lubricant, the effect of the polymer $M_{\mathrm{W}}$ on the dissolution kinetics upon exposure to laser radiation was investigated. Furthermore, the effect of the model drug on the dissolution kinetics was investigated using compacts containing $30 \mathrm{wt} \%$ of three different drugs (CCX, indomethacin (IND) and naproxen (NAP)), $69.25 \mathrm{wt} \%$ PVP12, $0.25 \mathrm{wt} \% \mathrm{PN}$ and $0.5 \mathrm{wt} \%$ lubricant. In perfect correlation to the Noyes-Whitney equation, this study showed that the use of PVP with the lowest viscosity, i.e., the lowest $M_{\mathrm{W}}$ (here PVP12), led to the fastest rate of amorphization compared to PVP17 and PVP25. Furthermore, NAP showed the fastest rate of amorphization, followed by IND and CCX in PVP12 due to its high solubility and small molecular size.
\end{abstract}

Keywords: in situ amorphization; near-IR laser radiation; amorphous solid dispersion; plasmonic photothermal nanoparticles; dissolution kinetics

\section{Introduction}

In situ amorphization describes the amorphization of a crystalline drug inside the final dosage form, such as a compact [1]. The amorphization can take place right after the manufacturing of the compact or directly before administration. In situ amorphization has been shown upon immersion in water, upon exposure to convectional heating and upon exposure to microwave and laser radiation [2-5]. Only using the latter two methods, complete amorphization has been obtained [4-6]. In order for a compact to absorb electromagnetic radiation, such as microwave or laser radiation, enabling excipients are necessary inside the compact [7-9].

In a recent study, photothermal silver plasmonic nanoaggregates (PNs) [10] were shown to be a promising enabling excipient for laser-induced in situ amorphization and 
were found to be non-toxic to Caco-2 cells at relevant doses. However, further studies are still needed to fully evaluate the toxicological profile of silver PNs associated with their oral administration [4]. Low amounts $(\leq 0.25 \mathrm{wt} \%)$ of PNs, which absorb light in the near-infrared region, have been shown to be sufficient to enable laser-induced in situ amorphization [4]. The approach was successfully utilized in a proof-of-concept study, in which complete amorphization of $30 \mathrm{wt} \%$ and $50 \mathrm{wt} \%$ of the model drug celecoxib (CCX) in the polymer polyvinylpyrrolidone (PVP12) was obtained upon exposure to laser radiation. The study showed that a faster rate of amorphization was obtained for compacts containing higher amounts of PNs $(0.25 \mathrm{wt} \%$ vs. $0.1 \mathrm{wt} \%)$, as well as for compacts exposed to laser radiation at higher laser intensities (for example, $1.31 \mathrm{~W} / \mathrm{cm}^{2}$ vs. $0.93 \mathrm{~W} / \mathrm{cm}^{2}$ ). However, the study was limited to the drug CCX and PVP12 [4].

PNs have a unique surface property, called localized surface plasmon, which allows the PNs to absorb radiation around their resonance frequency $[8,11]$. In this study, the PNs have been selected to absorb laser radiation in the near-infrared region [12,13]. Upon exposure to laser radiation, the PNs generate heat, which leads to a temperature increase inside the compact. Once temperatures above the glass transition temperature $\left(T_{\mathrm{g}}\right)$ of the polymer are reached, the drug readily dissolves into the mobile polymer and forms an amorphous solid dispersion (ASD) [14-16]. In previous studies, it was suggested that the rate of drug dissolution into the polymer only becomes significant when the compact temperature is above a certain temperature, labelled the temperature of the "onset" of the drug dissolution $\left(T_{\text {Onset }}\right)[4,14,15]$. As significant drug dissolution only occurs, when the polymer is mobile, i.e., at temperatures above the $T_{\mathrm{g}}$ of the polymer ( $\left.T_{\text {Onset }}>T_{\mathrm{g}}\right), T_{\text {Onset }}$ was defined as the temperature threshold, which needs to be surpassed upon exposure to laser radiation to achieve (measurable) in situ amorphization. Drug dissolution is kinetically limited at temperatures below the $T_{\mathrm{g}}$ of the polymer due to a high viscosity [14,17-19]. Furthermore, as in situ amorphization follows a dissolution process, which is a time- and temperature-dependent process [20,21], a certain temperature needs to be surpassed in the relatively short time-frame of laser exposure in order to obtain complete amorphization. With increasing drug dissolution into the mobile polymer, the viscosity of the polymer increases [22], which increases the temperature necessary to continue the dissolution process at the same dissolution speed and/or within the given time-frame. The temperature to (theoretically) obtain complete amorphization is labelled as the minimum temperature, $T_{\text {Min }}$, where $T_{\text {Min }}>T_{\text {Onset }}$. Both $T_{\text {Min }}$ and $T_{\text {Onset }}$ can be determined by thermal analysis (differential scanning calorimetry, DSC) for each drug-polymer combination and analyzing the dissolution endotherm of the obtained thermograms. The three temperatures, $T_{\mathrm{g}}, T_{\text {Onset }}$ and $T_{\mathrm{Min}}$, for the different compact compositions have been used to pre-assess which temperatures are necessary during exposure to laser radiation to achieve in situ amorphization.

The aim of the present study was two-fold: (i) to investigate the effect of the molecular weight $\left(M_{\mathrm{W}}\right)$ of the polymer PVP on the dissolution of the drug into the polymer and (ii) to investigate the effect of the model drug on the dissolution into the polymer PVP12.

For (i), $30 \mathrm{wt} \%$ of the model drug CCX was used together with PVP in different $M_{\mathrm{W}}$ grades, namely PVP12, PVP17 and PVP25. As the complete amorphization of CCX in PVP12 has been shown previously [4], it remains to be investigated how the rate and degree of amorphization of laser-induced in situ amorphization are affected using a higher $M_{\mathrm{W}}$ of PVP, i.e., the same polymer with a higher viscosity due to a higher $M_{\mathrm{w}}$. It is suggested based on studies investigating microwave-induced in situ amorphization that a higher viscosity due to a higher $M_{\mathrm{w}}$ will result in a slower rate and, therefore, an overall lower degree of amorphization upon exposure to radiation [22,23]. As the rate of amorphization is also dependent on the 'drug in polymer' solubility, it is important to mention that the solubility of CCX in PVP is independent of the $M_{\mathrm{W}}$ of PVP, i.e., CCX has the same solubility in the polymers PVP12, PVP17 and PVP25 [24]. The predicted solubility of CCX in PVP12 was previously reported at $41 \mathrm{wt} \%$ at $20{ }^{\circ} \mathrm{C}$ (confidence interval: $30-48 \mathrm{wt} \%$ ) [25]. This allows the investigation of the effect of the $M_{\mathrm{w}}$ of the polymer independently of the drug 
solubility in the polymer. The chosen drug load of $30 \mathrm{wt} \%$ is below the predicted solubility limit at room temperature; hence, all drugs should be able to dissolve into PVP under the experimental conditions.

For (ii), PVP12 was used with three different drugs, namely CCX, indomethacin (IND) and naproxen (NAP). All three drugs show solubility in PVP/PVP12 in the temperature range of the experimental conditions (i.e., from room temperature to the maximum temperature obtained during exposure to laser radiation). Furthermore, the three drugs are different in their glass-forming ability, i.e., NAP is a poor (class I), CCX a medium (class II) and IND a good glass-former (class III) [26] and have been previously used in the formation of ASD. Considering the drug molecules, it is not only the drug solubility in the polymer that has an effect on the dissolution rate, i.e., the rate of amorphization upon exposure to laser radiation, but also the molecular size of the drug molecule $[20,21]$. The molecular size of a drug molecule can be estimated in 2D by its minimum and maximum projected diameter. NAP has diameters of 1.1 and $0.5 \mathrm{~nm}$. The diameters of IND are 1.2 and $0.8 \mathrm{~nm}$, whilst CCX has diameters of 1.1 and $1.0 \mathrm{~nm}$. Hence, the molecular size of the drug molecules chosen for this study increase in the following order: NAP $<$ IND $<$ CCX. It is suggested, according to the Noyes-Whitney equation [20], that a smaller drug molecule will show enhanced dissolution kinetics, i.e., a higher rate of amorphization.

\section{Results and Discussion}

\subsection{The Effect of the Molecular Weight of Polyvinylpyrrolidone on In Situ Amorphization}

Using PVP12, PVP17 and PVP25, the influence of the $M_{\mathrm{W}}$ of PVP, i.e., the viscosity of the polymer, on the in situ amorphization of the drug CCX was investigated. It is commonly known that the viscosity and the $T_{\mathrm{g}}$ increase with the $M_{\mathrm{W}}$ of the polymer [27], which results in the following order for the increase in viscosity of PVP: PVP12 < PVP17 < PVP25. The viscosity of the polymer affects the dissolution kinetics of the drug into the mobile polymer, according to the diffusion coefficient of the Noyes-Whitney equation [20]. Furthermore, the increase in the $T_{\mathrm{g}}$ with increasing $M_{\mathrm{W}}$ of the polymer is suggested to result in an increase in $T_{\text {Onset }}$ and $T_{\text {Min }}$ of the compacts.

\subsubsection{The Determination of the Temperature Thresholds, $\mathrm{T}_{\text {Onset }}$ and $\mathrm{T}_{\text {Min }}$}

Prior to exposure to laser radiation, the $T_{\mathrm{g}}$ of the polymers, as well as the $T_{\text {Onset }}$ and $T_{\text {Min }}$, were determined by DSC. The results are shown in Table 1 . As can be seen, a higher $M_{\mathrm{W}}$ of the polymer resulted in a higher $T_{\mathrm{g}}$ of the polymer, which resulted in a higher $T_{\text {Onset }}$ and $T_{\text {Min }}$ for compacts containing the respective polymer. The temperature thresholds, $T_{\text {Onset }}$ and $T_{\text {Min }}$, were used as a pre-assessment to determine which temperatures were necessary to achieve (measurable) and complete in situ amorphization, respectively. Hence, a higher $M_{\mathrm{w}}$ of the polymer resulted in overall higher temperatures necessary to initiate the drug dissolution into the polymer and to obtain a fully amorphous ASD.

Table 1. The determination of the compact temperature thresholds, i.e., $T_{\text {Onset }}$ and $T_{\text {Min }}$, i.e., the temperatures where the in situ amorphization starts and is completed, respectively. $T_{\mathrm{g}} 1$ is the $T_{\mathrm{g}}$ of the bulk polymer containing water. $T_{\mathrm{g}} 2$ is the $T_{\mathrm{g}}$ of the water-free polymer. $T_{\mathrm{g}} 1$ and $T_{\mathrm{g}} 2$ are given as the Mean $\pm \mathrm{SD}(n=2)$.

\begin{tabular}{cccccc}
\hline Compact Composition & $\boldsymbol{T}_{\text {Onset }}\left[{ }^{\circ} \mathbf{C}\right]$ & $\boldsymbol{T}_{\text {Min }}\left[{ }^{\circ} \mathbf{C}\right]$ & Polymer & $\boldsymbol{T}_{\mathbf{g}} \mathbf{~ ( B u l k ) ~}\left[{ }^{\circ} \mathbf{C}\right]$ & $\boldsymbol{T}_{\mathbf{g}}(\mathbf{W a t e r}-\mathrm{Free})\left[{ }^{\circ} \mathbf{C}\right]$ \\
\hline $30 \mathrm{wt} \%$ CCX PVP12 & 103.6 & 132.2 & PVP12 & $39.7 \pm 0.2$ & $105.3 \pm 0.5$ \\
$30 \mathrm{wt} \%$ CCX PVP17 & 119.0 & 136.2 & PVP17 & $54.5 \pm 2.9$ & $120.6 \pm 0.1$ \\
$30 \mathrm{wt} \%$ CCX PVP25 & 127.8 & 145.4 & PVP25 & $56.1 \pm 0.6$ & $134.5 \pm 1.5$ \\
\hline $30 \mathrm{wt} \%$ IND PVP12 & 88.0 & 125.6 & PVP12 & $39.7 \pm 0.2$ & $105.3 \pm 0.5$ \\
$30 \mathrm{wt} \%$ NAP PVP12 & 74.5 & 100.5 & PVP12 & $39.7 \pm 0.2$ & $105.3 \pm 0.5$ \\
\hline
\end{tabular}

From Table 1, it can be seen that $T_{\text {Onset }}$ is a temperature between $T_{\mathrm{g}} 1$ and $T_{\mathrm{g}} 2$, i.e., a temperature where the polymer is mobile enough for the drug to dissolve in. The significant decrease in viscosity and thereby increase in mobility has previously been reported 
at approximately $T_{\mathrm{g}}+15-25^{\circ} \mathrm{C}$ (Note: $T_{\mathrm{g}}$ determined by differential scanning calorimetry; here $\left.T_{\mathrm{g}} 1\right)[4,14,17]$. As PVP contains water, which evaporates during exposure to laser radiation, the $T_{\mathrm{g}}$ will increase during exposure to laser radiation and will be between $T_{\mathrm{g}} 1$ and $T_{\mathrm{g}} 2$ during the exposure (not all water evaporates). PVP12, PVP17 and PVP25 contained $5.3 \pm 0.3 \mathrm{wt} \%, 9.5 \pm 0.3 \mathrm{wt} \%$ and $7.8 \pm 0.1 \mathrm{wt} \%$ water, respectively (mean $\pm \mathrm{SD}$, $n=3) . T_{\min }$ is the minimum temperature necessary to obtain a fully amorphous ASD during exposure to laser radiation. $T_{\min }$ is always at higher temperatures than $T_{\text {Onset }}$ and was found to be above $T_{\mathrm{g}} 2$ for CCX.

Based on the results of the thermal analysis, it was suggested that CCX would amorphize at a lower temperature and/or faster rate at the same temperature in compacts containing PVP12, followed by PVP17 and PVP25.

\subsubsection{The Rate of Amorphization upon Exposure to Laser Radiation}

In Figure 1, it can be seen that compacts containing CCX and PVP12 showed a faster rate of amorphization upon exposure to laser radiation than compacts containing PVP17. Compacts containing CCX and PVP12 became fully amorphous after $180 \mathrm{~s}$ of exposure to laser radiation, whilst compacts containing CCX and PVP17 required $210 \mathrm{~s}$ to become fully amorphous (Figure 1a,b). The relative residual crystallinity was determined by DSC analysis of the compacts before and after exposure to laser radiation. Complete amorphization was confirmed by XRPD (see Figure 2a-c).
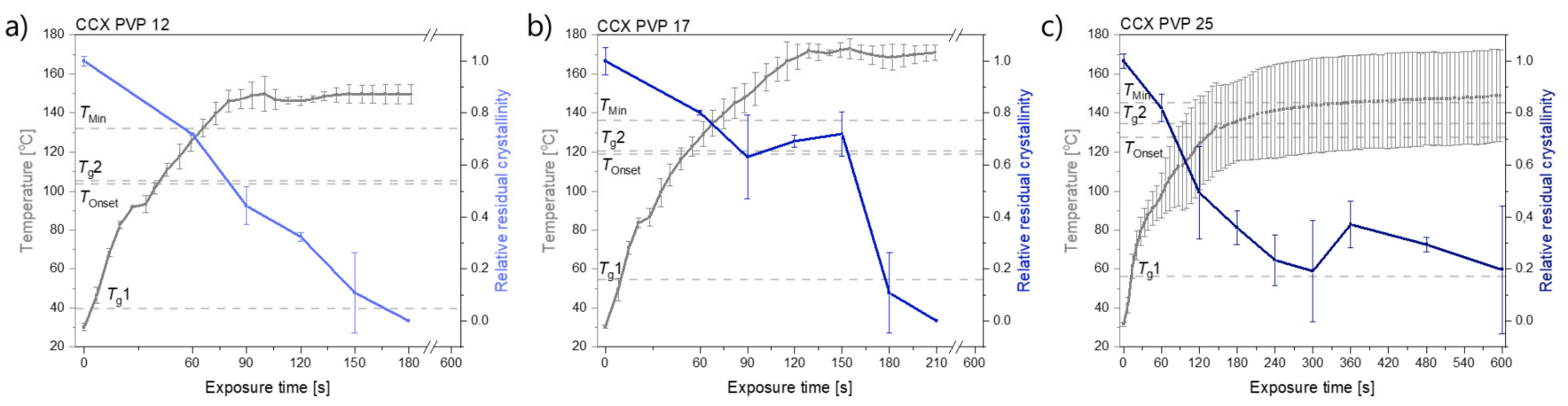

Figure 1. Temperature $\left[{ }^{\circ} \mathrm{C}\right]$ (left $y$-axis) and relative residual crystallinity (right $y$-axis, determined by DSC analysis) plotted as a function of exposure time [s]. All compacts were exposed at a laser intensity of $1.52 \mathrm{~W} / \mathrm{cm}^{2}$. (a) Compacts containing CCX and PVP12 (light blue), (b) Compacts containing CCX and PVP17 (blue), (c) Compacts containing CCX and PVP25 (dark blue). Mean $\pm \operatorname{SD}(n=3)$ for the left $y$-axis and mean $\pm \operatorname{SD}(n=2)$ for the right $y$-axis. The horizontal dashed lines indicate the temperatures, $T_{\mathrm{g}} 1, T_{\mathrm{g}} 2, T_{\text {Onset }}$ and $T_{\mathrm{Min}}$, as seen in Table 1.

Compacts containing CCX and PVP25 did not become fully amorphous. The temperatures obtained did not reach $T_{\mathrm{Min}}$ in any of the three investigated compacts to allow for complete amorphization, which can also be seen in the deviation of the results obtained for the relative residual crystallinity (Figure 1c). It is suggested that higher temperatures and/or longer exposure times would be necessary to achieve complete amorphization for compacts containing CCX and PVP25.

For compacts containing CCX and PVP12 or PVP17, the temperatures reached during exposure to laser radiation were above $T_{\mathrm{Min}}$, which means complete amorphization was possible at the obtained temperatures if sufficient time at this temperature was ensured. As the temperature thresholds, $T_{\text {Onset }}$ and $T_{\mathrm{Min}}$, were lower for compacts containing CCX and PVP12 compared to compacts containing PVP17, a faster rate of amorphization was obtained, even though higher maximum temperatures were obtained for compacts containing CCX and PVP17 $\left(149.6 \pm 4.9^{\circ} \mathrm{C}\right.$ (CCX/PVP12 compacts) vs. $171.0 \pm 3.8^{\circ} \mathrm{C}$ (CCX/PVP17 compacts); mean $\pm \mathrm{SD}(n=3))$. In both cases, the temperature increase was found to be similar in the first $90 \mathrm{sec}$ of exposure to laser radiation approximately until $T_{\text {Min }}$ was reached for compacts containing CCX and PVP12. It can be concluded that a polymer with a lower $M_{\mathrm{W}}$, and therefore a lower viscosity, resulted in an overall faster rate 
of amorphization compared to a higher $M_{\mathrm{w}}$ of the same polymer kind. These results are in line with previous results reported for microwave-induced in situ amorphization [22,23]. The results of this study also show that higher $M_{\mathrm{W}}$ of a polymer, e.g., PVP17, can be used for laser-induced in situ amorphization. This was not found for microwave-induced in situ amorphization, where PVP17 was not suitable to amorphize the same CCX-PVP17 combination. In a previous study, the compacts used for microwave-induced in situ amorphization contained $30 \mathrm{wt} \%$ CCX and PVP17 and were conditioned at high relative humidity to allow for water sorption. The sorbed water acted as a dielectric heating source and plasticizer, i.e., lowering the $T_{\mathrm{g}}$ of PVP17. During exposure of the compacts to microwave radiation, the sorbed water evaporated fast, which increased the $T_{\mathrm{g}}$ to a temperature that was not reachable during microwave irradiation, i.e., the amorphization came to a halt [23]. With the use of laser radiation instead of microwave radiation, polymers with a higher $M_{\mathrm{w}}$ and higher $T_{\mathrm{g}}$ become available for in situ amorphization, as it was shown that higher temperatures could be obtained.
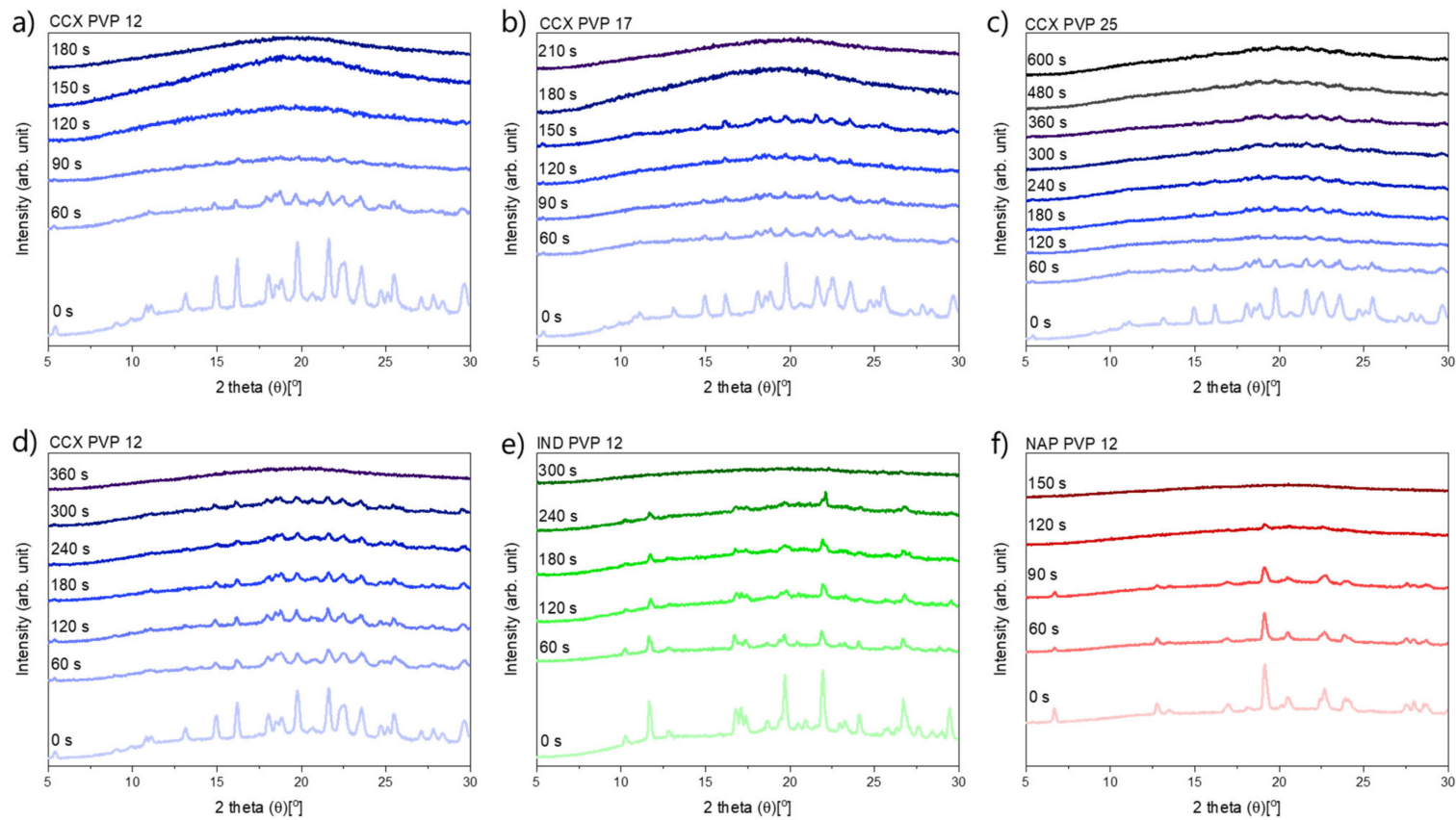

Figure 2. XRP-diffractograms at various exposure times. (a-c) exposed at a laser intensity of $1.52 \mathrm{~W} / \mathrm{cm}^{2},(\mathbf{a}) \mathrm{CCX}$ PVP12, (b) CCX PVP17, (c) CCX PVP25, (d-f) exposed at a lower laser intensity of $1.02 \mathrm{~W} / \mathrm{cm}^{2}$, (d) CCX PVP12, (e) IND PVP12, (f) NAP PVP12.

\subsection{The Influence of the Model Drug on In Situ Amorphization}

Using PVP12, the influence of the model drug on the in situ amorphization upon exposure to laser radiation was investigated. For this, CCX, IND and NAP were utilized.

\subsubsection{Solubility of Celecoxib, Indomethacin and Naproxen in Polyvinylpyrrolidone}

All model drugs show significant (predicted) solubility in PVP12 at room temperatures and the temperatures obtained during exposure to laser radiation. CCX, as mentioned above, has a predicted solubility of $41 \mathrm{wt} \%$ (confidence interval: $30-48 \mathrm{wt} \%$ ) at $20^{\circ} \mathrm{C}$ in PVP12. IND has a lower predicted solubility of $28 \mathrm{wt} \%$ (confidence interval: $14-38 \mathrm{wt} \%$ ) at $20^{\circ} \mathrm{C}$ [28]. The predicted solubility of NAP in PVP12 was determined to be at $61 \mathrm{wt} \%$ (confidence interval: $48-67 \mathrm{wt} \%$ ) at $20^{\circ} \mathrm{C}$ (see Figure 3). Hence, NAP has the highest predicted solubility in PVP12 at $20^{\circ} \mathrm{C}$, followed by CCX and IND. In the case of CCX and NAP, the chosen drug load of $30 \mathrm{wt} \%$ is below the predicted solubility limit at room temperature. For the drug IND, the chosen drug load of $30 \mathrm{wt} \%$ is in the confidence interval of the predicted solubility at room temperature. As the solubility increases with increasing 
temperature, a drug load of $30 \mathrm{wt} \%$ IND should be able to dissolve in PVP12 during the study. For example, at the maximum compact temperature $\left(129.9 \pm 2.4{ }^{\circ} \mathrm{C}\right), 64-76 \mathrm{wt} \%$ IND may, in theory, be soluble in PVP12.

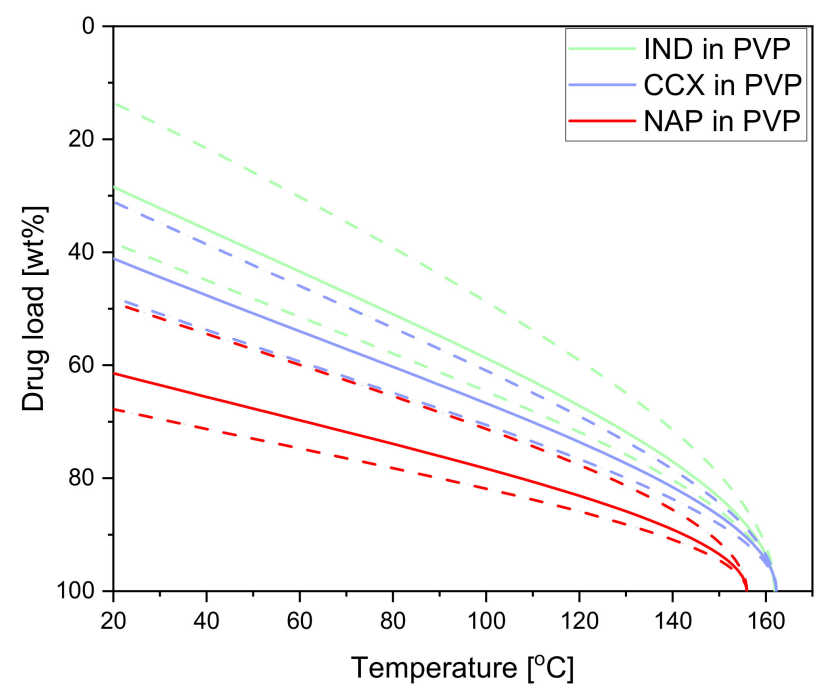

Figure 3. (Predicted) drug solubility curves for IND in PVP (green), CCX in PVP (blue) and NAP in PVP (red). Dashed lines indicate the confidence interval. The data for IND and CCX in PVP was taken from [28].

\subsubsection{The Determination of the Temperature Thresholds, $\mathrm{T}_{\text {Onset }}$ and $\mathrm{T}_{\text {Min }}$}

To pre-assess which temperatures (achieved upon exposure to laser radiation) were necessary to obtain complete in situ amorphization, the temperature thresholds of IND and NAP in PVP12 were also determined by DSC. As seen in Table 1, compacts containing CCX, IND, or NAP and PVP12 had different temperature thresholds. Even though the temperatures for the polymer $T_{\mathrm{g}} 1$ and $T_{\mathrm{g}} 2$ were identical, $T_{\text {Onset }}$ and $T_{\text {Min }}$ were different for each drug in PVP12. $T_{\text {Onset }}$ and $T_{\text {Min }}$ decreased in the following drug order: CCX $>$ IND $>$ NAP. Based on these results, it was suggested that compacts containing NAP and PVP12 would become fully amorphous at lower temperatures (and therefore following shorter exposure times) compared to compacts containing IND or CCX.

\subsubsection{The Rate of Amorphization upon Exposure to Laser Radiation}

Figure 4 shows the different compact temperatures and rates of amorphization obtained for compacts containing CCX, IND or NAP and PVP12 upon exposure to laser radiation.
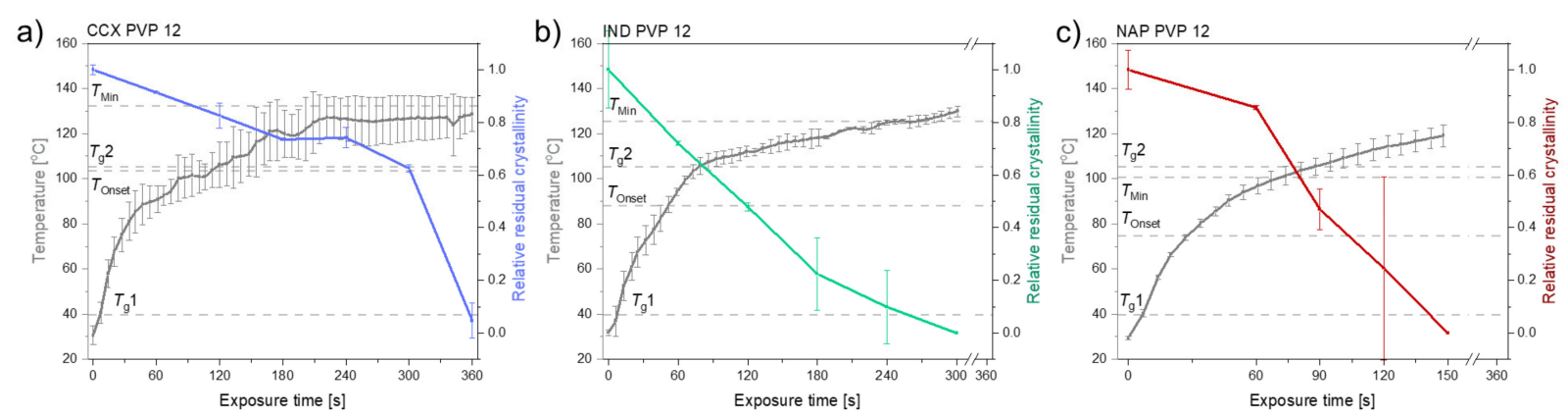

Figure 4. The temperature $\left({ }^{\circ} \mathrm{C}\right)$ (left $y$-axis) and relative residual crystallinity (right $y$-axis, determined by DSC analysis) plotted as a function of exposure time [s]. All compacts were exposed at a laser intensity of $1.02 \mathrm{~W} / \mathrm{cm}^{2}$. (a) Compacts containing CCX and PVP12 (light blue), (b) Compacts containing IND and PVP12 (light green), (c) Compacts containing $\mathrm{NAP}$ and PVP12 (red). Mean $\pm \mathrm{SD}(n=3)$ for the left $y$-axis and mean $\pm \mathrm{SD}(n=2)$ for the right $y$-axis. The horizontal dashed lines indicate the temperatures, $T_{\mathrm{g}} 1, T_{\mathrm{g}} 2, T_{\text {Onset }}$ and $T_{\mathrm{Min}}$, as seen in Table 1. 
It can be seen from Figure 4 that compacts containing NAP and PVP12 showed a faster rate of amorphization upon exposure to laser radiation than compacts containing IND or CCX. Compacts containing NAP and PVP12 became fully amorphous after $150 \mathrm{~s}$, whilst compacts containing IND or CCX and PVP12 were fully amorphous after $300 \mathrm{~s}$ and $360 \mathrm{~s}$, respectively (Figure 4). The complete amorphization of all compacts was confirmed by XRPD (see Figure 2d-f). For compacts containing NAP or IND, the compact temperatures reached were above $T_{\mathrm{Min}}$, and complete amorphization was obtained during exposure to laser radiation. For compacts containing CCX and PVP12, the compact temperatures reached during exposure to laser radiation were just below $T_{\mathrm{Min}}$. However, due to the differences in water content between the determination of $T_{\text {Min }}$ using DSC and the compact exposed to laser radiation, it is suggested that the theoretically calculated value $T_{\text {Min }}$ might be slightly overestimated, i.e., as shown above, complete amorphization was possible at temperatures just below the determined $T_{\text {Min }}$. As the temperature thresholds, $T_{\text {Onset }}$ and $T_{\text {Min }}$, were the lowest for compacts containing NAP as compared to compacts containing IND or CCX, a faster rate of amorphization was indeed obtained as suggested.

As the same polymer, and thereby the same viscosity, was used for this series of experiments, the rate of amorphization of the three model drugs into PVP 12 was only expected to be affected by the characteristics of the drugs. That means the dissolution of the three model drugs was affected by their solubility in the PVP12, their molecular sizes and their particle size, which affects the surface area available for dissolution, as described by the Noyes-Whitney equation (first-order dissolution kinetic) [20]. Due to the preparation method of the mixtures, similar particle sizes of the drugs (and PVP) can be expected, i.e., differences in the amorphization rate origin from the different solubilities in PVP12 and different molecular sizes of the drugs. However, the amorphization rate does not increase in the same order as seen for the drug-polymer solubilities, i.e., it is suggested that the molecular size of the drug dictated the amorphization rate in this study. Furthermore, the temperature thresholds determined follow the same order as the molecular size of the drug, e.g., NAP as the smallest molecule has the lowest temperature threshold, which also strongly indicates that the molecular size of the drug determined the amorphization rate.

It can be concluded that the use of compacts containing a drug with a moderate to high solubility in the polymer, low temperature thresholds and small molecular size resulted in an overall faster rate of amorphization compared to drugs with a lower solubility in the polymer, higher temperature thresholds and larger molecule sizes.

\subsection{Chemical Stability upon In Situ Amorphization}

As increased temperatures are obtained upon exposure to laser radiation, compacts exposed to the longest exposure times were tested for drug degradation using highperformance liquid chromatography (HPLC). No degradation was detected in any of the compacts (data not shown). As high temperatures are obtained during exposure to laser radiation, the thermal stability of the drugs (and polymers) is of great importance, i.e., possible degradation could alter the bioavailability and biological activity of certain thermosensitive drugs.

\section{Materials and Methods}

\subsection{Materials}

Celecoxib (CCX, $\left.M_{\mathrm{W}}=381.4 \mathrm{~g} / \mathrm{mol}\right)$, indomethacin $\left(\mathrm{IND}, M_{\mathrm{W}}=357.8 \mathrm{~g} / \mathrm{mol}\right)$, naproxen $\left(\mathrm{NAP}, M_{\mathrm{w}}=230.3 \mathrm{~g} / \mathrm{mol}\right)$ and magnesium stearate $\left(M_{\mathrm{w}}=591.3 \mathrm{~g} / \mathrm{mol}\right)$ were purchased from Fagron Nordic A/S (Copenhagen, Denmark). Kollidon ${ }^{\circledR} 12$ PF (polyvinylpyrrolidone (PVP), PVP12, $\left.M_{\mathrm{W}} \sim 2500 \mathrm{~g} / \mathrm{mol}\right), K_{\text {Kllidon }}{ }^{\circledR} 17$ PF (PVP17, $\left.M_{\mathrm{W}} \sim 9000 \mathrm{~g} / \mathrm{mol}\right)$ and Kollidon ${ }^{\circledR}$ 25 (PVP25, $M_{\mathrm{w}} \sim 24,000 \mathrm{~g} / \mathrm{mol}$ ) were a kind gift from BASF (Ludwigshafen, Germany).

Silver acetate (99.8\% anhydrous) was purchased from Alfa Aesar (Kandel, Germany). Hexamethyldisiloxane ( $\geq 98 \%$ ), acetonitrile (99.8\% anhydrous) and 2-ethylhexanoic acid (99\%) were purchased from Sigma-Aldrich (Stockholm, Sweden). The oxygen gas (flow: $5.0 \mathrm{~L} / \mathrm{min}$ ) for the flame-spray-pyrolysis synthesis was from Strandmøllen (Ljungby, Sweden). 
Ethanol (>99.7\%, HPLC grade) was purchased from VWR International (Leuven, Belgium). Acetonitrile (HPLC grade) and trifluoroacetic acid were purchased from SigmaAldrich (Søborg, Denmark). Purified water used for the mobile phase in the HPLC experiments was prepared using a MilliQ water system from LabWater (Los Angeles, CA, USA). All chemicals were used as received.

\subsection{Plasmonic Nanoaggregate Synthesis}

Silver PNs were obtained by flame-spray pyrolysis with a final composition of $98 \mathrm{wt} \%$ silver and $2 \mathrm{wt} \%$ silicon dioxide (spacer) [29], as introduced by Sotiriou et al. 2010 and 2011 [10,30] and Merkl et al. 2021 [12]. A detailed description of the procedure can be found in Hempel et al. (2021) [4].

\subsection{Compact Preparation}

Physical mixtures containing $30 \mathrm{wt} \%$ drug (CCX, IND or NAP), $69.25 \mathrm{wt} \%$ polymer (PVP12, PVP17 or PVP25), $0.5 \mathrm{wt} \%$ magnesium stearate (lubricant) and $0.25 \mathrm{wt} \%$ PN were obtained using mortar and pestle. A total of $50 \pm 2 \mathrm{mg}$ of each physical mixture was compressed into flat-faced compacts (diameter: $6 \mathrm{~mm}$ ) using an instrumented single-punch GTP-1 tablet machine from Gamlen Instruments (Nottingham, UK). The tablet machine was equipped with a $500 \mathrm{~kg}$ load cell (CT6-500-022) and used at a compaction pressure of $160 \mathrm{MPa}$.

\subsection{In Situ Amorphization}

In situ amorphization was induced using laser radiation at a wavelength of $805 \mathrm{~nm}$ (Near-infrared). The compacts were exposed to laser radiation for various exposure times, as indicated in Table 2. The maximum exposure time was set to $600 \mathrm{~s}$. The laser was a fiber-coupled laser diode, controlled using a Newport Laser Diode Controller Model 6000 (Mölndal, Sweden). The current to the laser diode was $4000 \mathrm{~mA}$, resulting in a laser power of $3321 \mathrm{~mW}$ ex fiber. No collimation lens was used at the fiber output, so the beam was diverging upon exiting the fiber, allowing for easy adjustment of laser intensity on the compact by varying the distance. The distance from the fiber tip to the bottom of the compact was either set to $6.75 \mathrm{~cm}$ or $8.25 \mathrm{~cm}$, which corresponds to a laser intensity of 1.52 or $1.02 \mathrm{~W} / \mathrm{cm}^{2}$, respectively. The two different laser intensities were used as at the high laser intensity, and the amorphization of compacts containing NAP and PVP12 was very fast, and the difference between IND and CCX compacts could not be determined, i.e., it was necessary to lower the laser intensity to investigate the influence of the drug in Section 2.2. At the low laser intensity, very long exposure times were necessary for compacts containing PVP17 and PVP25, exceeding $600 \mathrm{~s}$. Each compact was located on a cover glass slide and covered by another cover glass slide.

Table 2. Exposure times of compacts to laser radiation and the solid state of the drug determined by XRPD; c: residual crystallinity; a: fully amorphous. The experiments for the upper three rows were performed at a laser intensity of $1.52 \mathrm{~W} / \mathrm{cm}^{2}$. The experiments for the lower three rows were performed at a laser intensity of $1.02 \mathrm{~W} / \mathrm{cm}^{2}$.

\begin{tabular}{|c|c|c|c|c|c|c|c|c|c|c|c|}
\hline $\begin{array}{c}\text { Compact } \\
\text { Composition }\end{array}$ & $60 \mathrm{~s}$ & $90 \mathrm{~s}$ & $120 \mathrm{~s}$ & $150 \mathrm{~s}$ & $180 \mathrm{~s}$ & $210 \mathrm{~s}$ & $240 \mathrm{~s}$ & $300 \mathrm{~s}$ & $360 \mathrm{~s}$ & $480 \mathrm{~s}$ & $600 \mathrm{~s}$ \\
\hline CCX PVP12 & c & c & c & c & a & & & & & & \\
\hline CCX PVP17 & c & c & c & c & c & a & & & & & \\
\hline CCX PVP25 & c & & c & & c & & c & c & c & c & c \\
\hline CCX PVP12 & c & c & c & c & c & c & c & c & a & & \\
\hline IND PVP12 & c & c & c & c & c & c & c & a & & & \\
\hline NAP PVP12 & c & c & c & a & & & & & & & \\
\hline
\end{tabular}

During in situ amorphization, the surface temperature of the cover glass slide covering the compacts was measured using an infrared thermocamera testo 871 from Testo SE \& CO. KGaA (Lenzkirch, Germany). Using the thermography app, thermal images were 
approximately recorded every sixth sec and analyzed afterwards using the testo IRSoft Software (version 4.5). The thermal images were exported as .xlsx files and analyzed for the maximum temperature at each time point using Matlab R2019a (version 9.6.0.1335978) from Mathworks (Natick, MA, USA). Each experiment was conducted as a triplicate $(n=3)$.

\subsection{Qualitative Analysis of Crystallinity by Solid-State Analysis}

Solid-state analysis of the pure substances and the compacts before- and after exposure to laser radiation was performed using X-ray powder diffraction (XRPD). Prior to the analysis, the compacts were powdered using a mortar and pestle. The analysis was performed using an X'Pert Pro diffractometer from PANalytical (Eindhoven, The Netherlands) with $\mathrm{Cu} \mathrm{K} \alpha$ radiation $(\lambda=1.54187 \AA)$. The diffractograms were recorded from 5 to $30^{\circ} 2 \theta$ at $45 \mathrm{kV}$ and $40 \mathrm{~mA}$. The diffractograms were analyzed with the X'Pert HighScore Plus software (version 2.2.4.) from PANalytial (Eindhoven, The Netherlands).

\subsection{Thermal Analysis and Quantification of Crystallinity}

Thermal analysis was performed using a Q2000 DSC from TA Instruments (New Castle, DE, USA). All experiments were conducted under a nitrogen gas purge with a flow of $50 \mathrm{~mL} / \mathrm{min}$. The data was analyzed using the TRIOS software (version 5.1.1) from TA Instruments. The DSC cell constant was calibrated using indium.

\subsubsection{Quantification of the Residual Drug Crystallinity}

To quantify the residual crystallinity in compacts exposed to laser radiation, calibration curves were established for the drugs CCX, IND and NAP in PVP (PVP12, PVP17 and PVP25 for CCX and PVP12 for IND and NAP). Using a mortar and pestle, $100 \mathrm{mg}$ physical mixtures containing 10-30 wt \% drug (in $5 \mathrm{wt} \%$ increments) and 90-70 wt\% PVP $(12,17$ or 25) were obtained. Of each mixture, 3-5 mg was weighed into a Tzero aluminum pan and sealed with a perforated hermetic lid. A modulated DSC (mDSC) run was performed with a heating rate of $3^{\circ} \mathrm{C} / \mathrm{min}$ from $20^{\circ} \mathrm{C}$ to $180^{\circ} \mathrm{C}$. The modulation had an amplitude of $1^{\circ} \mathrm{C} / 50 \mathrm{~s}$. The dissolution enthalpy was determined in the total heat flow using the TA Instruments TRIOS software (version 5.1.1). Each calibration point was determined as a duplicate $(n=2)$. As the water evaporated upon heating, the sample mass was corrected by the water content of the pure polymer (see Section 3.7). The calibration curve was plotted and fitted with a polynomial function of the second degree with an intercept of 0 .

Compacts before -, and after exposure to laser radiation were carefully mortared, and $2-5 \mathrm{mg}$ of the powdered compacts were weighted into Tzero aluminum pans and sealed with a perforated hermetic lid. The dissolution enthalpy was determined using a modulated DSC (mDSC) run as described above. Each experiment was conducted as a duplicate $(n=2)$. The sample mass was also corrected by the water content (see Section 3.7). Using the established calibration curve, the residual crystallinity was calculated from the determined dissolution enthalpy.

\subsubsection{Determination of the (Predicted) Solubility of Naproxen in Polyvinylpyrrolidone}

For compacts containing NAP and PVP12, the drug-polymer solubility was determined. For this, $100 \mathrm{mg}$ physical mixtures were obtained by mortar and pestle with 70-90 wt \% NAP in $5 \mathrm{wt} \%$ increments. Afterwards, 3-5 mg of each mixture, and additionally the pure NAP, were weighed into Tzero aluminum pans and closed with a perforated hermetic lid. A temperature ramp of $1^{\circ} \mathrm{C} / \mathrm{min}$ to $180^{\circ} \mathrm{C}$ was applied. Each experiment was conducted as a duplicate $(n=2)$. The onset of melting was determined. Using the FloryHuggins approach [31,32], the solubility of the NAP in the PVP12 was calculated from the onset of the dissolution endotherm. Details of the method can be found in Knopp et al. (2015) [33]. 


\subsubsection{Glass Transition Temperatures of the Polymers}

The $T_{\mathrm{g}}$ of the polymers was also determined by thermal analysis. For each polymer, PVP12, PVP17 and PVP25, 3-5 mg was weighed into Tzero aluminum pans with hermetically sealing lids. In the case of determination of the water-free $T_{\mathrm{g}}\left(T_{\mathrm{g}} 2\right)$, the lid was perforated to allow the bulk water to evaporate. Each sample was first heated to $120^{\circ} \mathrm{C}$, followed by an isothermal period of $10 \mathrm{~min}$ before equilibrating to $20^{\circ} \mathrm{C}$. Subsequently, the sample was subjected to a modulated DSC (mDSC) run from 20 to $180^{\circ} \mathrm{C}$ at a heating rate of $3{ }^{\circ} \mathrm{C} / \mathrm{min}$ with an amplitude of $1^{\circ} \mathrm{C} / 50 \mathrm{~s}$. For the determination of the $T_{\mathrm{g}}$ with bulk-water $\left(T_{\mathrm{g}} 1\right)$, the sample was heated from 20 to $120^{\circ} \mathrm{C}$ using the above mDSC settings. The $T_{\mathrm{g}}$ was determined as the midpoint of the step change. Each experiment was conducted as a duplicate $(n=2)$.

3.6.4. Determination of the Temperature Thresholds, $\mathrm{T}_{\text {Onset }}$ and $\mathrm{T}_{\text {Min }}$

Using a mortar and pestle, $100 \mathrm{mg}$ physical mixtures containing $30 \mathrm{wt} \% \mathrm{drug}$, CCX, IND or NAP and $70 \mathrm{wt} \%$ PVP $(12,17,25$, see Table 2$)$ were obtained. Of each mixture, 3-5 $\mathrm{mg}$ was weighed into a Tzero aluminum pan and sealed with a perforated hermetic lid. A heating rate of $0.5^{\circ} \mathrm{C} / \mathrm{min}, 1{ }^{\circ} \mathrm{C} / \mathrm{min}, 2^{\circ} \mathrm{C} / \mathrm{min}, 3{ }^{\circ} \mathrm{C} / \mathrm{min}, 5^{\circ} \mathrm{C} / \mathrm{min}$ or $10^{\circ} \mathrm{C} / \mathrm{min}$ was applied from 20 to $180^{\circ} \mathrm{C}$. For each experiment, the on- and endset of the dissolution endotherm were determined. The values obtained for the different heating rates were extrapolated to a heating rate of $0{ }^{\circ} \mathrm{C} / \mathrm{min}$, which represents $T_{\text {Onset }}$ (for the onset of the dissolution at a heating rate of $0{ }^{\circ} \mathrm{C} / \mathrm{min}$ ) and $T_{\text {Min }}$ (for the endset of dissolution at a heating rate of $0{ }^{\circ} \mathrm{C} / \mathrm{min}$ ).

\subsection{Determination of the Water Content}

The water content of the polymers, PVP12, PVP17 and PVP25, the physical mixtures, and the compacts after exposure to laser radiation was determined using a Discovery thermogravimetric analyzer (TGA) from TA instruments Inc. (New Castle, DE, USA). The experiments were conducted under a nitrogen gas purge of $25 \mathrm{~mL} / \mathrm{min}$, and the weight loss was determined using the TA Instruments TRIOS software (version 5.1.1).

The water content of each sample was determined by heating of $10^{\circ} \mathrm{C} / \mathrm{min}$ from ambient temperature to $170{ }^{\circ} \mathrm{C}$. Each experiment for the pure compounds and the physical mixtures was conducted as a triplicate $(n=3)$ or duplicate $(n=2)$, respectively. The water content of the compacts exposed to laser radiation was determined for each exposure time and each compact composition as a single run $(n=1)$.

\subsection{Determination of the Drug Molecule Size}

The drug molecule size, i.e., the minimum and the maximum projected diameter, was determined using the Avogadro software, version 1.2.0. Avogadro is an open-source molecular builder and visualization tool: http:/ / avogadro.cc/ (accessed on 5 February 2021).

\subsection{Drug Degradation}

HPLC was used to quantify the amount of CCX, IND and NAP in the compacts before and after exposure to laser radiation, as well as to determine any signs of drug degradation. The experiments were performed using a 1260 Infinity HPCL from Agilent Technologies, Inc. (Santa Clara, CA, USA) on a reverse-phase Luna 5U C18(2) $100 \mathrm{~A}$ column with a length of $150 \mathrm{~mm}$ and a diameter of $4.60 \mu \mathrm{m}$ from Phenomenex Ltd. (Aschaffenburg, Germany). The HPLC analysis was performed at ambient temperature. The mobile phases were degassed before use (Note: trifluoroacetic acid was added after degassing of the purified water, as it would evaporate otherwise). Each sample was injected as a triplicate $(n=3)$.

The HPLC method for CCX was as follows: the mobile phase, purified water and ethanol, was eluted at a ratio of 3:7 $(v / v)$ with a flow rate of $1 \mathrm{~mL} / \mathrm{min}$. A sample volume of $10 \mu \mathrm{L}$ was injected, and UV-detection was performed at a wavelength of $251 \mathrm{~nm}$. The elution of CCX occurred at a retention time of $2.6 \mathrm{~min}$. 
The applied HPLC method for IND was adapted from Palmelund et al. (2019) [34]. Acetonitrile and purified water with $0.05 \%$ trifluoroacetic acid were used as the mobile phase in a 1:1 $(\mathrm{v} / \mathrm{v})$ ratio. The mobile phase was eluted at a flow rate of $1 \mathrm{~mL} / \mathrm{min}$, and a sample volume of $10 \mu \mathrm{L}$ was injected. UV detection was performed at a wavelength of $264 \mathrm{~nm}$. The retention time was found to be at $10.9 \mathrm{~min}$. The same HPLC method was applied for NAP, which was detected as a wavelength of $270 \mathrm{~nm}$ and a retention time of $5.5 \mathrm{~min}$.

To determine the drug content of the physical mixtures and compacts after the respective longest exposure time to laser radiation, HPLC samples were prepared by dissolving the physical mixtures or the mortared compacts in 50.0 or $100.0 \mathrm{~mL}$ of the organic phase of the respective mobile phase, i.e., ethanol in the case of CCX or acetonitrile for IND and NAP. Subsequently, the solution was filtered using a nylon syringe filter Q-max ${ }^{\circledR}$ RR $25 \mathrm{~mm}$ with a pore size of $0.45 \mu \mathrm{m}$ from Frisenette Aps (Knebel, Danmark), and the samples were analyzed by HPLC. The sample mass weight was corrected by the water content of the compact or mixture (see Section 3.7).

\section{Conclusions}

This study showed that for using PVP with different $M_{\mathrm{w}}$, a lower $M_{\mathrm{w}}$ is advantageous for the laser-induced in situ amorphization as it led to a faster rate of amorphization. The lower viscosity of PVP12 compared to PVP17 and PVP25 allowed the drug CCX to dissolve faster into the polymer during exposure to laser radiation, resulting in shorter exposure times until complete amorphization was reached. It is suggested that the same trend can be seen for other drugs or polymers. Using the same polymer, PVP12, but three different soluble model drugs, NAP, IND, and CCX, it could be shown that NAP resulted in the fastest rate of amorphization. The smaller molecular size of NAP, the lower temperature thresholds and the high solubility of NAP in PVP12 led to a faster rate of amorphization compared to compacts containing IND or CCX and PVP12.

Author Contributions: Conceptualization, N.-J.H., M.M.K., R.B. and K.L.; methodology, N.-J.H. and P.M.; formal analysis, N.-J.H.; investigation, N.-J.H. and A.K.H.; data curation, N.-J.H.; writingoriginal draft preparation, N.-J.H.; writing-review and editing, N.-J.H., P.M., M.M.K., R.B., A.T., A.K.H., G.A.S. and K.L.; visualization, N.-J.H.; supervision, M.M.K., R.B., A.T., G.A.S. and K.L.; project administration, K.L.; funding acquisition, N.-J.H., A.T., G.A.S. and K.L. All authors have read and agreed to the published version of the manuscript.

Funding: All authors gratefully acknowledge funding from NordForsk (Nordic University Hub project No. 85352; Nordic POP). The authors N.H., M.M.K., R.B. and K.L., would like to thank the Independent Research Fund Denmark for financial support (Grant No. DFF-7026-00052B). G.A.S. acknowledges funding received from the European Research Council (ERC) under the European Union's Horizon 2020 research and innovation program (ERC Grant agreement No. 758705), the Swedish Foundation for Strategic Research (FFL18-0043) and from the Swedish Research Council (2016-03471). The Science for Life Laboratory is gratefully acknowledged for financial support by A.T.

Institutional Review Board Statement: Not applicable.

Informed Consent Statement: Not applicable.

Data Availability Statement: The raw data is available from the authors upon request.

Acknowledgments: Ole Bjarlin Jensen from DTU Fotonik is acknowledged for loaning the laser equipment.

Conflicts of Interest: The authors declare no conflict of interest.

Sample Availability: Samples are not available. 


\section{References}

1. Qiang, W.; Lobmann, K.; McCoy, C.P.; Andrews, G.P.; Zhao, M. Microwave-Induced In Situ Amorphization: A New Strategy for Tackling the Stability Issue of Amorphous Solid Dispersions. Pharmaceutics 2020, 12, 655. [CrossRef]

2. Priemel, P.A.; Laitinen, R.; Grohganz, H.; Rades, T.; Strachan, C.J. In situ amorphisation of indomethacin with Eudragit(R) E during dissolution. Eur. J. Pharm. BioPharm. 2013, 85, 1259-1265. [CrossRef]

3. Hempel, N.J.; Knopp, M.M.; Berthelsen, R.; Lobmann, K. Convection-Induced vs. Microwave Radiation-Induced in situ Drug Amorphization. Molecules 2020, 25, 1068. [CrossRef]

4. Hempel, N.J.; Merkl, P.; Asad, S.; Knopp, M.M.; Berthelsen, R.; Bergstrom, C.A.S.; Teleki, A.; Sotiriou, G.A.; Lobmann, K. Utilizing Laser Activation of Photothermal Plasmonic Nanoparticles to Induce On-Demand Drug Amorphization inside a Tablet. Mol. Pharm. 2021. [CrossRef]

5. Holm, T.P.; Knopp, M.M.; Lobmann, K.; Berthelsen, R. Microwave induced in situ amorphisation facilitated by crystalline hydrates. Eur. J. Pharm. Sci. 2021, 163, 105858. [CrossRef]

6. Hempel, N.J.; Knopp, M.M.; Berthelsen, R.; Zeitler, J.A.; Lobmann, K. The influence of drug and polymer particle size on the in situ amorphization using microwave irradiation. Eur. J. Pharm. BioPharm. 2020, 149, 77-84. [CrossRef]

7. Grant, E.; Halstead, B.J. Dielectric parameters relevant to microwave dielectric heating. Chem. Soc. Rev. 1998, 27, 213-224. [CrossRef]

8. Kravets, V.G.; Kabashin, A.V.; Barnes, W.L.; Grigorenko, A.N. Plasmonic Surface Lattice Resonances: A Review of Properties and Applications. Chem. Rev. 2018, 118, 5912-5951. [CrossRef]

9. Kim, M.; Lee, J.H.; Nam, J.M. Plasmonic Photothermal Nanoparticles for Biomedical Applications. Adv. Sci. $2019,6,1900471$. [CrossRef]

10. Sotiriou, G.A.; Pratsinis, S.E. Antibacterial activity of nanosilver ions and particles. Environ. Sci. Technol. 2010, 44, 5649-5654. [CrossRef]

11. Boken, J.; Khurana, P.; Thatai, S.; Kumar, D.; Prasad, S. Plasmonic nanoparticles and their analytical applications: A review. Appl. Spectrosc. Rev. 2017, 52, 774-820. [CrossRef]

12. Merkl, P.; Zhou, S.; Zaganiaris, A.; Shahata, M.; Eleftheraki, A.; Thersleff, T.; Sotiriou, G.A. Plasmonic Coupling in Silver Nanoparticle Aggregates and Their Polymer Composite Films for Near-Infrared Photothermal Biofilm Eradication. ACS Appl. Nano Mater. 2021. [CrossRef]

13. Sotiriou, G.A.; Blattmann, C.O.; Deligiannakis, Y. Nanoantioxidant-driven plasmon enhanced proton-coupled electron transfer. Nanoscale 2016, 8, 796-803. [CrossRef]

14. Moseson, D.E.; Taylor, L.S. The application of temperature-composition phase diagrams for hot melt extrusion processing of amorphous solid dispersions to prevent residual crystallinity. Int. J. Pharm. 2018, 553, 454-466. [CrossRef]

15. Moseson, D.E.; Parker, A.S.; Gilpin, C.J.; Stewart, A.A.; Beaudoin, S.P.; Taylor, L.S. Dissolution of Indomethacin Crystals into a Polymer Melt: Role of Diffusion and Fragmentation. Cryst. Growth Des. 2019, 19, 3315-3328. [CrossRef]

16. Tian, Y.; Caron, V.; Jones, D.S.; Healy, A.-M.; Andrews, G.P. Using Flory-Huggins phase diagrams as a pre-formulation tool for the production of amorphous solid dispersions: A comparison between hot-melt extrusion and spray drying. J. Pharm. Pharmacol. 2014, 66, 256-274. [CrossRef]

17. Roudaut, G.; Simatos, D.; Champion, D.; Contreras-Lopez, E.; Le Meste, M. Molecular mobility around the glass transition temperature: A mini review. Innov. Food Sci. Emerg. Technol. 2004, 5, 127-134. [CrossRef]

18. Hancock, B.C.; Shamblin, S.L.; Zografi, G. Molecular Mobility of Amorphous Pharmaceutical Solids Below Their Glass Transition Temperatures. Pharm. Res. 1995, 12, 799-806. [CrossRef]

19. Hilden, L.R.; Morris, K.R. Physics of amorphous solids. J. Pharm. Sci. 2004, 93, 3-12. [CrossRef]

20. Noyes, A.A.; Whitney, W.R. The Rate of Solution of Solid Substances in Their Own Solutions. J. Am. Chem. Soc. 2002, 19, 930-934. [CrossRef]

21. Edward, J.T. Molecular volumes and the Stokes-Einstein equation. J. Chem. Educ. 1970, 47, 261. [CrossRef]

22. Hempel, N.J.; Dao, T.; Knopp, M.M.; Berthelsen, R.; Lobmann, K. The Influence of Temperature and Viscosity of Polyethylene Glycol on the Rate of Microwave-Induced In Situ Amorphization of Celecoxib. Molecules 2020, 26, 110. [CrossRef]

23. Doreth, M.; Lobmann, K.; Priemel, P.; Grohganz, H.; Taylor, R.; Holm, R.; Lopez de Diego, H.; Rades, T. Influence of PVP molecular weight on the microwave assisted in situ amorphization of indomethacin. Eur. J. Pharm. BioPharm. 2018, 122, 62-69. [CrossRef]

24. Knopp, M.M.; Olesen, N.E.; Holm, P.; Langguth, P.; Holm, R.; Rades, T. Influence of Polymer Molecular Weight on Drug-Polymer Solubility: A Comparison between Experimentally Determined Solubility in PVP and Prediction Derived from Solubility in Monomer. J. Pharm. Sci. 2015, 104, 2905-2912. [CrossRef]

25. Knopp, M.M.; Gannon, N.; Porsch, I.; Rask, M.B.; Olesen, N.E.; Langguth, P.; Holm, R.; Rades, T. A Promising New Method to Estimate Drug-Polymer Solubility at Room Temperature. J. Pharm. Sci. 2016, 105, 2621-2624. [CrossRef]

26. Baird, J.A.; Van Eerdenbrugh, B.; Taylor, L.S. A Classification System to Assess the Crystallization Tendency of Organic Molecules from Undercooled Melts. J. Pharm. Sci. 2010, 99, 3787-3806. [CrossRef]

27. Nunes, R.W.; Martin, J.R.; Johnson, J.F. Influence of molecular weight and molecular weight distribution on mechanical properties of polymers. Polym. Eng. Sci. 1982, 22, 205-228. 
28. Knopp, M.M.; Olesen, N.E.; Holm, P.; Lobmann, K.; Holm, R.; Langguth, P.; Rades, T. Evaluation of drug-polymer solubility curves through formal statistical analysis: Comparison of preparation techniques. J. Pharm. Sci. 2015, 104, 44-51. [CrossRef]

29. Mädler, L.; Kammler, H.K.; Mueller, R.; Pratsinis, S.E. Controlled synthesis of nanostructured particles by flame spray pyrolysis. J. Aerosol. Sci. 2002, 33, 369-389. [CrossRef]

30. Sotiriou, G.A.; Teleki, A.; Camenzind, A.; Krumeich, F.; Meyer, A.; Panke, S.; Pratsinis, S.E. Nanosilver on nanostructured silica: Antibacterial activity and Ag surface area. Chem. Eng. J. 2011, 170, 547-554. [CrossRef]

31. Marsac, P.J.; Shamblin, S.L.; Taylor, L.S. Theoretical and practical approaches for prediction of drug-polymer miscibility and solubility. Pharm. Res. 2006, 23, 2417-2426. [CrossRef]

32. Marsac, P.J.; Li, T.; Taylor, L.S. Estimation of drug-polymer miscibility and solubility in amorphous solid dispersions using experimentally determined interaction parameters. Pharm. Res. 2009, 26, 139-151. [CrossRef]

33. Knopp, M.M.; Tajber, L.; Tian, Y.; Olesen, N.E.; Jones, D.S.; Kozyra, A.; Löbmann, K.; Paluch, K.; Brennan, C.M.; Holm, R.; et al. Comparative Study of Different Methods for the Prediction of Drug-Polymer Solubility. Mol. Pharm. 2015, 12, 3408-3419. [CrossRef]

34. Palmelund, H.; Andersson, M.P.; Asgreen, C.J.; Boyd, B.J.; Rantanen, J.; Lobmann, K. Tailor-made solvents for pharmaceutical use? Experimental and computational approach for determining solubility in deep eutectic solvents (DES). Int. J. Pharm. X 2019, 1, 100034. [CrossRef] 